\title{
Intravenous Tissue Plasminogen Activator Therapy for Ischemic Stroke Patients who Caused Car Accidents
}

\author{
Ichiro Deguchi' ${ }^{*}$, Takeshi Hayashi' ${ }^{1}$, Manabu Nemoto² and Norio Tanahashi ${ }^{1}$
}

${ }^{1}$ Department of Neurology, Saitama Medical University, International Medical Center, Japan

${ }^{2}$ Department of Emergency Medicine, Saitama Medical University, International Medical Center, Japan

\begin{abstract}
Stroke can cause car accidents. In our hospital, intravenous tissue plasminogen activator (t-PA) therapy was given to three ischemic stroke patients who had caused car accidents. Since stroke was suspected based on clinical manifestations on arrival, head examinations, as well as detailed examinations of the whole body, were performed immediately. After confirming the absence of hemorrhage from traumatic injuries, the patients were treated with intravenous t-PA. No patients had adverse events including hemorrhage. With extensive examinations, thrombolytic therapy can be safely given to ischemic stroke patients involved in traffic accidents.
\end{abstract}

Keywords: Car accidents; Tissue plasminogen activator therapy; Ischemic stroke

\section{Introduction}

Stroke can cause car accidents. About $0.1 \%$ of traffic accidents are caused by stroke [1]. In most motor vehicle accidents, the precise time of occurrence is known, and therefore, the time of stroke onset can be easily identified. Since most such patients are brought to hospital by ambulance, they are often good candidates for intravenous t-PA (tissue plasminogen activator) therapy. However, they tend not to undergo t-PA therapy because of concern about complications including hemorrhage. However, as far as we know, there have been no reports on intravenous t-PA therapy for ischemic stroke patients who had caused car accidents. Three ischemic stroke patients who safely underwent intravenous t-PA therapy following car accidents are reported.

\section{Case 1}

A 68-year-old man with a history of hypertension experienced sudden weakness of the right side of the body while driving a car. Although he tried to push on the brake pedal, he was unable to do so, and the car crashed into a pole. He was transferred to our hospital 46 min after the accident. On arrival, the patient was fully conscious, but dysarthria and right hemiparesis were confirmed. His National Institute of Health Stroke Scale (NIHSS) score was 6. There were no abnormal findings in the chest and abdomen and no bruises anywhere on the body. Although brain computed tomography (CT) scanning showed no abnormality, magnetic resonance imaging (MRI) revealed an acute ischemic stroke in the left frontal and parietal lobes (Figure 1A). Magnetic resonance angiography (MRA) showed occlusion of the M2 segment of the left middle cerebral artery (Figure 1B). Radiographic examinations including CT of the chest, abdomen, and pelvis, as well as Focused Assessment with Sonography for Trauma (FAST), excluded accident-related traumatic injuries. Since the patient had no contraindications for intravenous t-PA therapy, t-PA was administered intravenously $155 \mathrm{~min}$ after stroke onset. No adverse events related to t-PA infusion were observed. His neurological symptoms improved, and he was discharged home on the 17th day, with a modified Rankin scale (mRS) score of 1.

\section{Case 2}

The patient was a 70-year-old man. The car that he was driving made a sudden turn and collided with a wall. A person who was present at the accident site called an ambulance, and he was transported to our hospital $45 \mathrm{~min}$ after the accident. He had a history of stroke and paroxysmal atrial fibrillation (he was on no antithrombotic medication). On arrival, he had a slight disturbance of consciousness. Neurologic examinations revealed right conjugate ocular deviation,
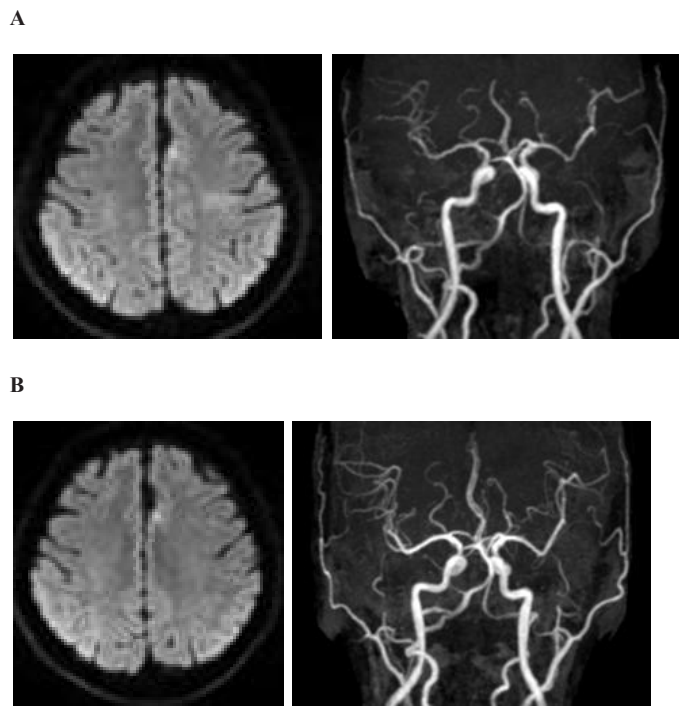

Figure 1: MRI (DWI) and MRA on arrival and 24 hours after tissue plasminogen activator infusion. A: DWI shows high intensity in the left frontal and parietal lobes, and MRA shows left MCA (M2) occlusion. B: Recanalization of the left MCA (M2)

*Corresponding author: Ichiro Deguchi, Saitama Medical University, International Medical Center, Hidaka, Saitama, Japan, Tel: +81-42-984-4359; Fax: +81-42-9840664; E-mail: ideguchi5221@yahoo.co.jp

Received February 12, 2013; Accepted March 27, 2013; Published April 01, 2013

Citation: Deguchi I, Hayashi T, Nemoto M, Tanahashi N (2013) Intravenous Tissue Plasminogen Activator Therapy for Ischemic Stroke Patients who Caused Car Accidents. J Neurol Neurophysiol 4: 145. doi:10.4172/2155-9562.1000145

Copyright: @ 2013 Deguchi I, et al. This is an open-access article distributed under the terms of the Creative Commons Attribution License, which permits unrestricted use, distribution, and reproduction in any medium, provided the original author and source are credited. 
Citation: Deguchi I, Hayashi T, Nemoto M, Tanahashi N (2013) Intravenous Tissue Plasminogen Activator Therapy for Ischemic Stroke Patients who Caused Car Accidents. J Neurol Neurophysiol 4: 145. doi:10.4172/2155-9562.1000145

dysarthria, and left hemiparesis. His NIHSS score was 12 . There were no abnormal findings in the chest or abdomen, and no bruises were observed anywhere on the body. A brain CT scan showed a blurred corticomedullary junction of the right frontal lobe. Head MRI revealed an acute ischemic stroke in the right frontal and parietal lobes (Figure 2A). MRA showed occlusion of the right anterior and middle cerebral arteries. Radiographic examinations including CT of the chest, abdomen, and pelvis, as well as FAST, excluded accidentrelated traumatic injuries (Figure 2B). Since the patient had no contraindications for intravenous t-PA, it was administered $150 \mathrm{~min}$ after stroke onset. Since t-PA administration did not improve his symptoms, additional cerebral endovascular treatment was given. He had no adverse events related to the t-PA infusions, and he was transferred to another hospital on the 26th day, with a mRS score of 3.

\section{Case 3}

A 69-year-old man suddenly became unable to maintain a conversation while driving a car and veered into oncoming traffic. His car made contact with another one, and he was transferred to our hospital 70 min after the accident. He had a history of hypertension, hyperlipidemia, and diabetes. On arrival, his Glasgow Coma Scale was E-4, V-1, and M-5. Neurologic examinations revealed that the patient had left conjugate ocular deviation, dysarthria, right hemiparesis, and aphasia. His NIHSS score was 15 . There were no abnormal findings in the chest or abdomen and no bruises anywhere on the body. A brain CT scan showed early ischemic lesions in the left frontal lobe. Brain MRI revealed acute ischemic stroke in the left frontal, temporal, and parietal lobes (Figure 3A). MRA showed occlusion of the left middle cerebral artery. Radiographic examinations including CT of the chest, abdomen, and pelvis, as well as FAST, excluded accident-related traumatic injuries (Figure 3B). Since the patient had no contraindications for intravenous t-PA infusion, it was administered 175 min after stroke onset. He did not experience any adverse events related to the t-PA

A
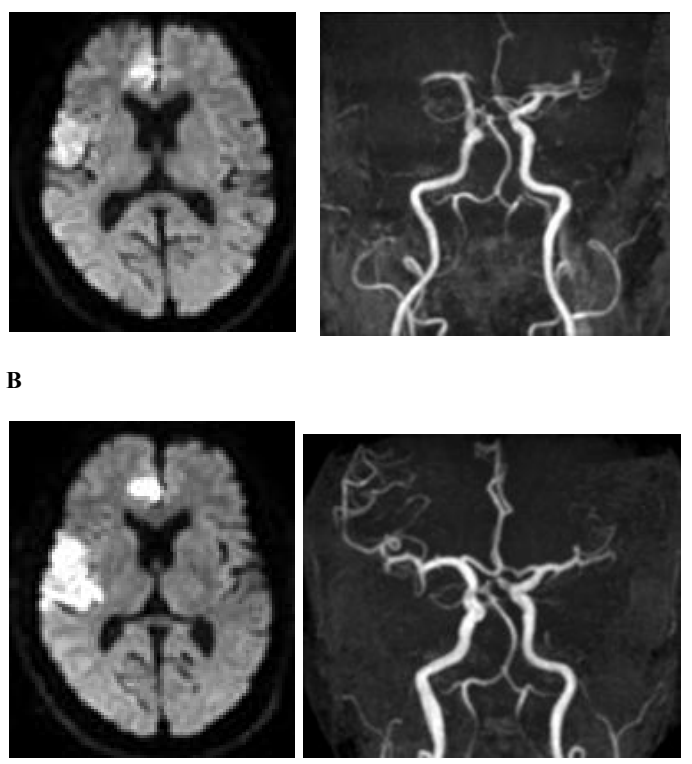

Figure 2: MRI (DWI) and MRA on arrival and 24 hours after tissue plasminogen activator infusion. A: DWI shows high intensity in the right frontal and parietal lobes, and MRA shows right ACA and MCA (M1) occlusions. $\mathrm{B}$ : Recanalization of the right ACA and MCA (M1).

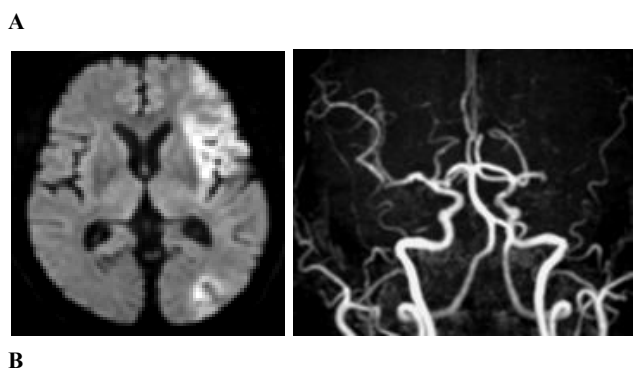

B
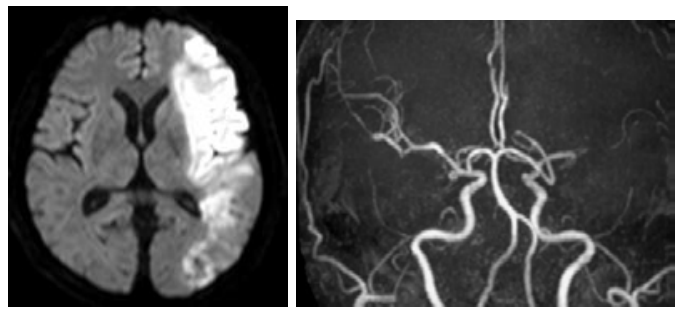

Figure 3: MRI (DWI) and MRA on arrival and 24 hours after tissue plasminogen activator infusion. A: DWI shows high intensity in the left frontal, temporal, and parietal lobes, and MRA shows left MCA (M1) occlusion. $\mathrm{B}$ : No recanalization of the left MCA is seen (M1).

infusions, and he was transferred to another hospital on the 48th day, with a mRS score of 5 .

\section{Discussion}

The most important aspect of treating acute ischemic stroke patients involved in car accidents with intravenous t-PA therapy is the assessment of traumatic injuries. Overlooked intracranial or extracranial hemorrhages may result in serious consequences. Accurate assessment of patients with traumatic injuries may be difficult in facilities where no emergency physicians specialized in trauma are available. For treatment with t-PA infusions, assessment of the patients would further be difficult because the therapeutic time window for intravenous t-PA therapy is limited to 4.5 hours after ischemic stroke onset. Our cooperative approach with emergency physicians and stroke team staff led to the safe administration of intravenous t-PA therapy in the above cases. Moreover, none of these patients had serious trauma, a contraindication for intravenous t-PA [2]. In most cases of ischemic stroke, the degree of impairment of consciousness is mild, and the patients are often able to take actions to avoid danger, resulting in mild traumatic injuries $[3,4]$.

An issue that needs attention is the difficulty of obtaining information from patients with impaired consciousness or aphasia. In such cases, patients are unable to explain their symptoms, and assessment of trauma and confirmation of contraindications for t-PA are difficult. It is also difficult to confirm whether stroke preceded or resulted from car accident in some cases. In Case 3, the patient experienced aphasia and was unable to provide a medical history or describe symptom progression. However, his wife, who was also in the car, gave us detailed information which allowed us to perform intravenous t-PA therapy.

Traumatic injuries and hemorrhagic complications might be a concern, but thorough examinations enable physicians to safely perform t-PA therapy even in stroke patients who caused traffic accidents. Cooperation between emergency physicians and neurologists, which could save ischemic stroke patients, is very important. 
Citation: Deguchi I, Hayashi T, Nemoto M, Tanahashi N (2013) Intravenous Tissue Plasminogen Activator Therapy for Ischemic Stroke Patients who Caused Car Accidents. J Neurol Neurophysiol 4: 145. doi:10.4172/2155-9562.1000145

\section{Reference}

1. Finelli PF, Lee N (1996) Stroke and automobile accidents. Conn med 60: 145147

2. Shinohara Y, Ogawa A, Suzuki N, Katayama Y, Kimura A (2009) Japanese Guidelines for the Management of Stroke 2009.
3. Foulkes MA, Wolf PA, Price TR, Mohr JP, Hier DB (1988) The Stroke Data Bank: design, methods, and baseline characteristics. Stroke 19: 547-554.

4. Bogousslavsky J, Van Melle G, Regli F (1988) The Lausanne Stroke Registry: analysis of 1,000 consecutive patients with first stroke. Stroke 19: 1083-1092. 